\title{
Bioanalysis: the best of 2009
}

\author{
“...we thought we would try to encourage further excellence and perhaps a little friendly competition by selecting \\ a "Best Article of the Year."
}

As we look back on our first year, we are particularly impressed with how quickly Bioanalysis has become a real force in this field. In personal interactions, many people have congratulated us, or complimented the journal and the quality of articles, the layout and the void it fills in the field of drug, metabolite and biomarker analysis. Truthfully, we cannot claim responsibility or credit for any of these wonderful attributes. They are the outcomes of the great work and thoughts of you, our audience and contributors!

So, we thought we would try to encourage further excellence and perhaps a little friendly competition by selecting a 'Best Article of the Year'. To begin with, we individually present our shortlist of candidates and our selection process.

\section{Brian}

There are essentially two types of articles to choose from - original research articles and reviews. In our first year, we had more of the latter, as one might expect. There were many excellent articles touching on many salient portions of a number of analytical issues. My definition of a good review article is one that describes the main issues, uses examples, and places the issue in the context of drug development (or the field in which it is an issue). "Multiplexed antibody arrays for the discovery and validation of glycosylated protein biomarkers" [1] and "Metabolomics for early detection of drug-induced kidney injury: review of the current status" [2] are a couple of good examples.

However, I also have to admit my bias: I am partial to original research articles. These manuscripts describe efforts to further our knowledge and make improvements to what we do. They are not as comprehensive as the review articles, and sometimes seem fairly 'small' in comparison, but they represent the future of this discipline.
- Criteria for selecting pharmacokinetic repeat study samples

The authors discuss pharmacokinetic 'anomalies' and the criteria used to repeat the analysis of certain samples. Technically, it describes nothing new analytically, but the article highlights the importance of bioanalysis and how misunderstanding analytical method validation can have a big impact on study outcomes in ways that might not have been foreseen [3].

\section{- Detection of doping with recombinant human growth hormone}

This was an interesting article to me because it describes many of the complex issues associated with measuring a moving target accurately, in a more routine, real-life setting (if you can call the Olympics a routine, real-life setting) compared with drug development. The authors did a nice job describing the physiology of human growth hormone, how it is impacted by environmental influences and the strengths and limitations of the analytical methodologies [4].

\section{- Assessing the matrix effects of} hemolyzed samples in bioanalysis

I liked this article because it is an attempt to address a widely recognized, but as yet unresolved, issue. Like the couterion issue for anticoagulants, this topic appears to be one that can be prospectively studied and resolved. This article is a big step in that direction, with the authors identifying several aspects of interference and providing several solutions for these problems [5].

\section{- Impact of plasma \& whole-blood} anticoagulant couterion choice on drug stability and matrix effects during bioanalysis Again, this article describes efforts to better understand a ubiquitous issue in method validation. In this case, the authors examined one of the

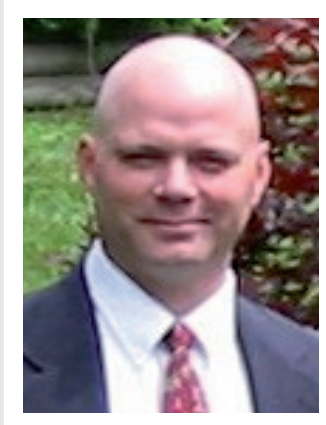

Brian Booth ${ }^{\dagger *}$

${ }^{\dagger}$ Author for correspondence: Senior Editor, Bioanalysis,

Division of Clinical Pharmacology V,

Office of Clinical Pharmacology

Center for Drug Research and

Evaluation Research, US FDA, Silver

Spring, MD 20993-002, USA

Tel.: + I 3017961508

E-mail: brian.booth@fda.hhs.gov

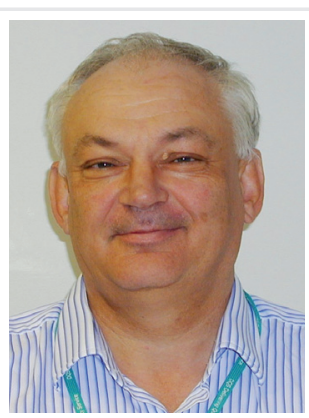

Howard Hill*

Senior Editor, Bioanalysis,

Huntingdon Life Sciences, Woolley

Road, Alconbury, Huntingdon,

Cambridgeshire,

PEI6 5HS, UK

E-mail: hillh

@ukorg.huntingdon.com

*Contributed equally to this article.

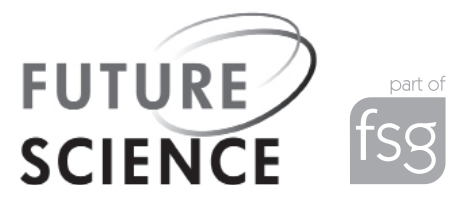


potential causes for a difference between counterions. This was a good effort to understand the science behind the phenomenon [6].

Bioanalysis of siRNA and oligonucleotide therapeutics in biological fluids and tissues

This review was of interest because of the difficulties we have seen in measuring plasma concentrations of these types of molecules. The authors did a nice job describing the chemistry of these entities and the methods used to measure them [7].

\section{Howard}

At the risk of appearing a little contrary, I have taken a slightly different approach to Brian in that I have tried to 'equalize' all publication types (i.e., reviews, regulatory focus and original papers) using some personal criteria. I looked for papers that added value to the bioanalytical community by providing new knowledge or new insights into old issues, or by collating information that helps to provide new insight or new directions. In summary, I carried out an objective evaluation of these papers, each of which, in their own way, contribute to making the bioanalyst's life a little easier, or issues understandable - thereby providing a way forward.

Not mentioned but figured greatly in my selection process were reviews related to glycobioanalysis, such as "Nano-LC and HPLCchip-ESI-MS: an emerging technique for glycobioanalysis" [8] and "Multiplexed antibody arrays for the discovery and validation of glycosylated protein biomarkers" [1] above. Of the last 15 I narrowed it down to, nine were macromolecule related but only one got to the final six. This was not a reflection of the scientific content nor of the work in developing and evaluating many of the review topics. It reflects the broader appeal of the final six that I choose. We have both developed personal approaches to selecting the 'best' paper, which hopefully reflects the flexibility of content and appeal of the journal.

- Assessing the matrix effects of hemolyzed samples in bioanalysis

This is a topic that is frequently raised and usually conveniently ignored. How do you (conveniently and easily) measure the level of hemolysis and relate it to the 'postulated' matrix effect. There may be other ways to measure the extent of hemolysis but this paper does illustrate the intuitive thought that a small amount of hemolysis goes a long way (in terms of color intensity) and, apparently, in terms of ion suppression.
It might be interesting to see what effect the correlation between the extent of hemolysis (at lower levels) has on these assays in particular. A useful paper in terms of tips and a sobering reminder of the impact that hemolysis can have on results [5].

Ligand-binding assays: risk of using a platform supported by a single vendor

Although this is a review article it nevertheless records the author's experiences with the discontinuation of the BioVeris ${ }^{\mathrm{TM}}$ platform. This phenomenon, and the associated financial and regulatory risks, can have a major impact on the extent and type of innovative techniques that are brought to market, possibly resulting in the loss or delay in implementing a revolutionary technology. The author succinctly discusses these issues and provides food for thought for those wishing to tread new paths [9].

\section{Pharmacokinetics application of} microdevices \& microsampling techniques

The development of dried blood spots as a potential 'microdevice' has raised the awareness of the need to implement the 3Rs (replacement, refinement and reduction) in preclinical research. This article provides an excellent comparison of the different technologies and hopefully raises the awareness of what is available, the need for such technologies and the hope (and impetus) that some of these technologies can be improved and perfected. While the direct inline use of biosensors provides the ultimate in cost reduction, speed and adherence to the $3 R$ s, it is perhaps the furthest off, but this does not mean it is a less worthy goal [10].

\section{High-throughput salting-out assisted} liquid-liquid extraction \& ultrafast LC for same-day delivery of first-in-human bioanalytical data

This is a neat combination of an old well-established and almost well-forgotten phenomenon - salting out in liquid-liquid extraction with the relatively new concept of ultrafast LC for solving an old need. It enables the bioanalyst to cost effectively turn around data in a regulatory compliant manner prior to escalating to the next dose [11].

\section{Metabolites in safety testing}

This article provides regulatory insights on a topic that has been high on bioanalyst's agendas for the last 10 years, albeit caveated by the usual US FDA disclaimer, and is coupled 
with useful illustrations and examples. It succinctly combines the scientific issues with the related regulatory documents driving this concern and, as such, provides a helpful digestible overview with useful references [12].

\section{- Matrix effect elimination} during LC-MS/MS bioanalytical method development

Another hot and enduring topic, the authors, in addition to this review, have made significant contributions to this field and have provided both a useful historical context (don't reinvent the wheel) with a review of the issues and practical procedures that can be used to resolve, minimize or manage this phenomenon [13].

\section{And the Winner is...}

As you can see, it was a difficult choice given the many high-quality examples we published. In the end, we have chosen the Bioanalytical Challenge paper "Assessing the matrix effects of hemolyzed samples in bioanalysis" by Nicola Hughes, Navgeet Bajaj, Juan Fan and Ernest Wong of Biovail (Toronto, Canada) as the Best Bioanalysis Article of 2009 [5]. Congratulations!

The paper by Hughes et al. is a Bioanalytical Challenge article, which are designed to act as an educational resource by providing insights into practical laboratory issues. In these articles, we encourage authors to describe both laboratory successes and failures, so that other researchers can learn from their experiences.

We would like to share this excellent article with our readers so, in addition to presenting the authors with a complimentary 1-year subscription, the article is now available to download for free until the end of September (2 months from publication date).

We would like to close by thanking all of our authors and readers; we look forward to making similar difficult decisions in 2010!

\section{Disclaimer}

The views expressed are those of Brian Booth and do not reflect official policy of the US FDA. No official endorsement by the FDA is intended or should be inferred.

\section{Financial \& competing interests disclosure}

The authors have no relevant affiliations or financial involvement with any organization or entity with a financial interest in or financial conflict with the subject matter or materials discussed in the manuscript. This includes employment, consultancies, honoraria, stock ownership or options, expert testimony, grants or patents received or pending, or royalties.

No writing assistance was utilized in the production of this manuscript.

\section{Bibliography}

1 Nelson BP. Multiplexed antibody arrays for the discovery and validation of glycosylated protein biomarkers. Bioanalysis 1(8), 1431-1444 (2009).

2 Boudonck KJ, Rose DJ, Karoly ED, Lee DP, Lawton KA, Lapinskas PJ. Metabolomics for early detection of drug-induced kidney injury: review of the current status. Bioanalysis 1(9), 1645-1663 (2009).

3 King PS, Aungst BJ, Shen H-SL. Criteria for selecting pharmacokinetic repeat study samples. Bioanalysis 1(8), 1415-1418 (2009).

$4 \mathrm{He}$ C, Wu M. Detection of doping with recombinant human growth hormone. Bioanalysis 1(5), 953-965 (2009).
5 Hughes NC, Bajaj N, Fan J, Wong EYK. Assessing the matrix effects of hemolyzed samples in bioanalysis. Bioanalysis 1(6), 1057-1066 (2009).

6 Bergeron M, Bergeron A, Furtado M, Garofolo F. Impact of plasma and wholeblood anticoagulant counter ion choice on drug stability and matrix effects during bioanalysis. Bioanalysis 1(3), 537-548 (2009).

7 Tremblay GA, Oldfield PR. Bioanalysis of siRNA and oligonucleotide therapeutics in biological fluids and tissues. Bioanalysis 1(3), 595-609 (2009).

8 Kirsch S, Bindila L. Nano-LC and HPLCchip-ESI-MS: an emerging technique for glycobioanalysis. Bioanalysis 1(7), 1307-1327 (2009).
9 Yohrling J. Ligand-binding assays: risk of using a platform supported by a single vendor. Bioanalysis 1(3), 629-636 (2009).

10 Musteata MF. Pharmacokinetic application of microdevices and microsampling techniques. Bioanalysis 1(1), 171-185 (2009).

11 Zhang J, Myasein F, Rodila R, Wu H, El-Shourbagy TA. High-throughput salting-out assisted liquid/liquid extraction and ultrafast LC for same-day delivery of first-in-human bioanalytical data. Bioanalysis 1(4), 715-719 (2009).

12 Robison TW, Jacobs A. Metabolites in safety testing. Bioanalysis 1(7), 1193-1200 (2009).

13 Côté C, Bergeron A, Mess J-N, Furtado M, Garofolo F. Matrix effect elimination during LC-MS/MS bioanalytical method development. Bioanalysis 1(7), 1243-1257 (2009). 